\title{
Isolated Single Umbilical Artery: Implications For
}

\section{Pregnancy}

\author{
Mehmet Nafi Sakar ${ }^{1}$, Süleyman Cemil Oğlak ${ }^{2^{\star}}$, Süreyya Demir ${ }^{3}$, Hüseyin Gültekin ${ }^{4}$, Bülent Demir ${ }^{5}$ \\ ${ }^{1}$ Department of Obstetrics and Gynecology, Memorial Diyarbakir Hospital, Diyarbakir \\ ${ }^{2}$ Department of Obstetrics and Gynecology, Health Sciences University, Gaz̧i Yaşargil Training and Research Hospital, \\ Diyarbakir, Turkey \\ ${ }^{3}$ Department of Obstetrics and Gynecology, Faculty of Medicine, Dokuz. Eylül University, Izmir, Turkey \\ ${ }^{4}$ Department of Obstetrics and Gynecology, Suleymaniye Maternity Education and Research Hospital, Istanbul, Turkey \\ ${ }^{5}$ Department of Obstetrics and Gynecology, Faculty of Medicine, Canakkale Onsekiz. Mart University, Çanakkale, \\ Turkey
}

\begin{abstract}
This study aimed to evaluate whether the presence of an isolated single umbilical artery (iSUA) is associated with adverse perinatal outcomes.

Fifty-one patients with iSUA and 54 controls were enrolled in the case-control study. Demographic characteristics and perinatal outcomes were recorded.

All patients had normal umbilical artery (UMA) Doppler findings, and their de tailed ultrasound weeks were between 18 and 23 weeks. The birth weights of the babies in the iSUA group were significantly lower than the control group $(2898.82 \pm 434.56 \mathrm{~g}$ vs $3143.70 \pm 419.09 \mathrm{~g}, \mathrm{p}=0.004)$. In the iSUA group, oligohydramnios $(7.8 \% \mathrm{vs} 0 \%)$, preterm premature rupture of membranes (PPROM) (3.9\% vs $0 \%$ ), preterm delivery (9.8\% vs 3.7\%), small for gestational age (SGA) (11.8\% vs $3.7 \%)$ and neonatal intensive care unit (NICU) (2\% vs $0 \%)$ admission rates were higher than control group, but there was no statistical significance $(\mathrm{p}>0.05)$. But the total adverse pregnancy outcomes were observed in $35.3 \%(18 / 51)$ of cases with iSUA is higher than controls $7.4 \%(4 / 54)(p=0.001)$.

In our study, iSUA is found to be associated with low newborn weight. In the iSUA group, oligohydramnios, PPROM, preterm delivery, SGA, and NICU admission rates were high, but there was no statistical significance. But the total adverse pregnancy outcomes were higher in the iSUA group. These results indicate that close follow-up is essential to prevent and manage adverse perinatal outcomes in iSUA patients.
\end{abstract}

Key Words: Pregnancy, isolated single umbilical artery, perinatal outcomes

\section{Introduction}

The development of the blood vessels and fetal circulation begins by the fourth week of embryonic life. At the commencement of pregnancy, the umbilical cord involves two umbilical arteries and two umbilical veins that the right umbilical vein regresses. Consequently, the umbilical cord consists of two arteries and one vein enclosed by Wharton gel. The umbilical artery pair carrying the deoxygenated blood is drained into the placenta's chorionic vessels by passing through the connecting stalk, which will then become the umbilical cord (1).

A single umbilical artery (SUA) is described as the absence of one of the two umbilical arteries. SUA is the most frequently occurring abnormality of the umbilical cord (2). The frequency of SUA is $0,3-1 \%(3,4)$. The exact pathogenesis of SUA is still unclear, yet there are two potential explanations: primary agenesis or thrombotic atrophy of one umbilical artery (5). The majority of umbilical cord abnormalities can be recognized by ultrasound (6). Umbilical cord abnormalities are typically associated with fetal chromosomal, structural abnormalities, and adverse perinatal outcomes (7-9).

When SUA is detected, it is necessary to exclude possible pathological conditions by using a detailed ultrasonographic (US) evaluation in the second trimester. SUA is called isolated SUA (iSUA) in the lack of extra structural and chromosomal abnormalities. $65-80 \%$ of SUA cases are isolated (10).

\footnotetext{
*Corresponding Author: Süleyman Cemil Oğlak, University of Health Sciences, Gazi Yaşargil Training and Research Hospital, Diyarbakır, Turkey 
Previous studies have identified numerous possible risk factors for iSUA, such as maternal age, ethnicity, multiparity, maternal smoking, and multiple pregnancies (11-14). However, there is not enough evidence to confirm these results.

iSUA is related to adverse perinatal outcomes including oligohydramnios, preterm premature rupture of membranes (PPROM), preterm birth, small for gestational age (SGA), and low placental weight. Postnatal studies have shown that iSUA pregnancies are significantly lower in birth weight compared to control group pregnancies $(11,15,16,17)$.

In the current study, we aimed to evaluate whether an isolated single umbilical artery (iSUA) is associated with adverse perinatal outcomes.

\section{Materials and Methods}

Fifty-one patients with iSUA and fifty-four controls were enrolled in the retrospective casecontrol study at the Department of Obstetrics and Gynecology, Suleymaniye Maternity Education and Research Hospital, Health Sciences University, Istanbul Turkey; Department of Obstetrics and Gynecology, Diyarbakir Gazi Yasargil Education and Research Hospital, and the Health Sciences University in Diyarbakir, Turkey between March 2010 and February 2018. The ethical review committee of Gazi Yasargil Training and Research Hospital approved the study. The patients found to have two-vessel umbilical cords without any additional structural or chromosomal abnormalities were selected. Participants in control groups were single pregnancy with the normal three-vessel cord and without any additional structural or chromosomal abnormalities. Gestational age was measured by the first day of the woman's last menstrual period and/or first-trimester ultrasound measurements. We diagnosed SUA in the second trimester when a cross-sectional picture of the umbilical cord proved only two vessels and/or in the oblique transverse section using color flow mapping to envision one of two umbilical arteries at nearby to the fetal bladder. A detailed ultrasonographic examination was performed in all fetuses with SUA by an experienced ultrasonographer or perinatologist. All patients underwent prenatal screening tests. However, fetal karyotyping was not performed because there is no evidence of an increased risk of chromosomal abnormalities in iSUA cases in prenatal screening tests (18).

We defined oligohydramnios as an amniotic fluid index $\leq 5 \mathrm{~cm}$. The preterm premature rupture of membranes (PPROM) was defined as amniotic fluid leakage before the 37 th week. We described preterm delivery as delivery before the 37 th week. We defined small for gestational age (SGA) as birth weight less than the gestational weeks 10 th percentile. The total adverse pregnancy outcomes were defined as the presence of at least one of the following: oligohydramnios, preterm premature rupture of membrane, preterm delivery, SGA, and neonatal intensive care unit admission.

Delivery mode, gender, newborn weight, and 1 st and 5th minute Apgar scores were noted. Demographic characteristics and ultrasonographic findings were pointed out in the first examination. We obtained pregnancy outcomes from hospital documentation. We confirmed the diagnosis of SUA by macroscopic examination of the newborn. Paediatrician performed a physical exam of all babies.

Statistical Analysis: IBM SPSS 21.0 for Windows (SPSS Inc., Chicago, IL, USA) statistical package program was used for statistical evaluation of our research data. Shapiro Wilks test was used to determine whether the numerical data matched the normality distribution. Student's t-test was used to compare quantitative data between groups and the Mann-Whitney $U$ test was used to compare nonnormal distribution parameters. The chi-square test, Fisher's exact test and Yates's continuity correction were used to compare the qualitative data. Significance was evaluated at $\mathrm{P}$-value $<0.05$.

\section{Results}

The mean maternal age was $26.51 \pm 3.54$ in the iSUA group and $26.98 \pm 4.33$ in the control group. There was no statistically significant difference between the groups in terms of median age and parity numbers $(\mathrm{p}>0.05$; Table 1$)$. All patients had normal umbilical artery (UMA), and Doppler screenings were made between 18 and 23 weeks (Table 1). There was no significant difference between groups in terms of delivery type, gestational age, gender, APGAR scores $(p>0.05$, Table 2). None of the prenatal screening tests of patients with iSUA had a high risk for a chromosomal abnormality, and no patient was advised to undergo invasive prenatal tests, including amniocentesis and cordocentesis.

The birth weights of the babies in the iSUA group were significantly lower than the control group $(2898.82 \pm 434.56 \mathrm{~g}$ and $3143.70 \pm 419.09 \mathrm{~g}$, respectively, $\mathrm{p}=0.004$, Table 2 ). 
Table 1. Maternal and antenatal demographics in the iSUA and Controls groups

\begin{tabular}{llll}
\hline Parameters & iSUA & Controls & p-value \\
& n:51 & n:54 & $26.98 \pm 4.33$ \\
\hline Maternal age, mean \pm sd & $26.51 \pm 3.54$ & 0.544 \\
Parity, median (min-max) & $1(0-3)$ & $0(0-4)$ & 20.306 \\
Detailed ultrasound week & $18-23$ weeks & $18-23$ weeks & - \\
\hline
\end{tabular}

${ }^{1}$ Student's t-test

${ }^{2}$ Mann-Whitney U Test

Table 2. Perinatal outcomes in the iSUA and Controls groups

\begin{tabular}{|c|c|c|c|}
\hline Parameters & $\begin{array}{c}\text { iSUA } \\
\mathrm{n}: 51\end{array}$ & $\begin{array}{l}\text { Controls } \\
\mathrm{n}: 54\end{array}$ & p-value \\
\hline $\begin{array}{l}\text { Gestational age at delivery (weeks), } \\
\text { mean } \pm \text { sd }\end{array}$ & $38.21 \pm 2.01$ & $38.80 \pm 1.17$ & 10.077 \\
\hline \multicolumn{4}{|l|}{ Delivery mode n (\%) } \\
\hline Vaginal & $20(39.2 \%)$ & $30(55.6 \%)$ & 20.894 \\
\hline Cesarean sectio & $31(60.8 \%)$ & $24(44.4 \%)$ & \\
\hline \multicolumn{4}{|l|}{ APGAR score, median (min-max) } \\
\hline 1.minute & $8(6-9)$ & $8(7-9)$ & 30.861 \\
\hline 5. minute & $10(7-10)$ & $9(8-10)$ & 30.183 \\
\hline \multicolumn{4}{|l|}{ Gendern $(\%)$} \\
\hline Female & $30(58.8 \%)$ & $29(53.7 \%)$ & 20.597 \\
\hline Male & $21(41.2 \%)$ & $25(46.3 \%)$ & \\
\hline Newborn weight (g), mean \pm sd & $2898.82 \pm 434.56$ & $3143.70 \pm 419.09$ & $10.004 *$ \\
\hline \multicolumn{4}{|l|}{ Perinatal outcomesn $(\%)$} \\
\hline Oligohydramniosis & $4(7.8 \%)$ & $0(0 \%)$ & ${ }^{4} 0.052$ \\
\hline Preterm premature rupture of membrane & $2(3.9 \%)$ & $0(0 \%)$ & ${ }^{4} 0.234$ \\
\hline Preterm delivery & $5(9.8 \%)$ & $2(3.7 \%)$ & ${ }^{4} 0.261$ \\
\hline Small for gestational age & $6(11.8 \%)$ & $2(3.7 \%)$ & ${ }^{4} 0.153$ \\
\hline Neonatal intensive care unit admission & $1(2 \%)$ & $0(0 \%)$ & ${ }^{4} 0.486$ \\
\hline Total adverse perinatal outcomes & $18(35.3 \%)$ & $4(7.4 \%)$ & $50.001 *$ \\
\hline
\end{tabular}

${ }^{1}$ Student's t-test

${ }^{2}$ Chi-squared Test

${ }^{3}$ Mann-Whitney U Test

${ }^{4}$ Fisher's Exact Test

${ }^{5}$ Yates' continuity correction

$* \mathrm{p}<0.05$

The incidence of oligohydramnios in infants within the iSUA group $(7.8 \%)$ was higher than that in the control group $(0 \%)$, but this difference was not statistically significant ( $\mathrm{p}=0.052$; Table 2$)$. PPROM, preterm birth, SGA and neonatal intensive care unit (NICU) admission rates were high in the iSUA group, but there was no significant statistical difference ( $p>0.05$, Table 2$)$. Nevertheless, the total adverse pregnancy outcomes observed in $35.3 \%(18 / 51)$ of cases with iSUA were higher than in $7.4 \%(4 / 54)$ of the control group ( $\mathrm{p}=0.001$; Table 2$)$.

\section{Discussion}

In our study, the birth weights of the neonates in the iSUA group $((2898.82 \pm 434.56$ g) were significantly lower than the control group (3143.70 $\pm 419.09 \mathrm{~g}, \mathrm{p}=0.004)$. In the iSUA group, oligohydramnios $(7.8 \%$ vs $0 \%)$, preterm premature rupture of membranes (PPROM) $(3.9 \%$ vs $0 \%$ ), preterm delivery $(9.8 \%$ vs $3.7 \%$ ), small for gestational age (SGA) $(11.8 \%$ vs $3.7 \%)$ and neonatal intensive care unit (NICU) $(2 \%$ vs $0 \%$ ) admission rates were higher than control group, 
but there was no statistical significance $(\mathrm{p}>0.05)$. However, the total adverse pregnancy outcomes were observed in $35.3 \%(18 / 51)$ of cases with iSUA is higher than in $7.4 \%(4 / 54)$ of controls $(\mathrm{p}=0.001)$.

SUA patients with additional congenital anomalies have a high risk of adverse perinatal outcomes. However, it remains unclear in iSUA cases. In numerous studies, iSUA has been associated with oligohydramnios, polyhydramnios, preterm birth, PPROM, SGA, low placental weight, gestational diabetes mellitus (GDM) and perinatal mortality (19-21).

$\mathrm{Mu}$ et al. investigated the perinatal outcomes of asymptomatic iSUA patients. They were stated that the sample size was comparatively small in their study. Still, the incidence of SGA was significantly higher in iSUA patients $(35 \%)$ than the control group $(3.6 \%, \mathrm{p}=0.011)$ (21). In a study Horton et al., the birth weight of iSUA neonates was significantly lower than the control group $(3279 \pm 404 \mathrm{~g}$, and $3423 \pm 374 \mathrm{~g}$, respectively, $\mathrm{p}=0.016)(12)$. Also, the duration in the NICU was significantly longer in iSUA group (1.25 \pm 2.2 days) than the controls $(0.48 \pm 1.25$ days, $\mathrm{p}=0.023)$ (12). Numerous studies confirmed that SUA is associated with an increased risk of preterm labor $(2,13,15,17)$.

In our study, birth weight was low in iSUA patients. Preterm delivery, PPROM, oligohydramnios, and NICU hospitalization rates increased but were not statistically significant. However, the total adverse pregnancy outcomes observed in $35.3 \%(18 / 51)$ of cases with iSUA is higher than in $7.4 \%(4 / 54)$ of the control group $(\mathrm{p}=0.001)$.

In some studies, iSUA was reported as no increased risk for preterm birth $(13,14)$, intrauterine growth restriction (IUGR) (22), SGA, or prenatal morbidity and mortality (3).

A study performed by Blum et al. (23) found increases in preterm birth, cord prolapse, placental abruption, non-reassuring fetal heart rates and cesarean birth rates in the iSUA group. Neonatal birth weight was significantly lower in the iSUA group (1988.0 \pm 697$)$ than the control group (2388.3 $\pm 481, \mathrm{p}<0.001) .1 \mathrm{st}$ and 5 th minute APGAR scores were significantly lower. Perinatal mortality was found to be significantly higher in the iSUA group. In the iSUA group, preterm delivery was individually associated with perinatal mortality (23). Naveiro-Fuentes et al. showed increased cesarean section rate, low birth weight scores, and they reported altered cord $\mathrm{pH}$ values in the direction of acidosis in their studies (24).

Our study supports these studies' findings in terms of significant low birth weight in the iSUA group. However, there was no significant difference detected in terms of the delivery type, or $1 \mathrm{st}$ and 5 th minute APGAR scores. The results of our study were similar to those of Chett Jonn et al. and Ashwal et al., who did not find any correlation between iSUA and APGAR scores $(11,25)$.

A study by Burshtein et al. showed that iSUA patients (38.3 \pm 3.0 weeks) are related to one week earlier delivery when compared to patients without SUA $(39.3 \pm 2.1$ weeks, $\mathrm{p}<0.001)$ (17). However, in our study, we did not observe any significant difference between the two groups regarding the gestational week on delivery.

Increased NICU hospitalization rates, pregnancyinduced hypertension (PIH), and perinatal mortality rates have been reported in iSUA cases $(10,17)$. The presence of ISUA was associated with a more than five times increased risk of intrauterine and perinatal death and with placental and cord complications (26).

In our study, one of the neonates needed NICU hospitalization due to the early preterm delivery. We did not encounter adverse outcomes such as perinatal mortality and PIH.

In one study, they reported an increased risk of renal anomalies in iSUA cases, but most of them were minor and self-limited (27). Therefore, extensive urologic radiographic studies are not recommended in asymptomatic newborns unless there are additional risk factors for malformations. In our study, no study was performed on renal anomalies since the newborns were asymptomatic.

In most studies and meta-analyzes, iSUA has been associated with increased adverse perinatal outcomes. In line with this evidence, iSUA cases should be evaluated as a high-risk pregnancy and followed up closely.

The incidence of chromosomal abnormalities is scarce in iSUA patients. Dagklis et al. stated an incidence of $0.7 \%$ in iSUA cases, yet found no evidence for an increased risk of chromosomal abnormalities in overt iSUA cases (18). We performed prenatal screening test in all cases, yet since there is no evidence for karyotyping in iSUA cases, it was not included in our study.

Joó et al. reported a male to female ratio of $1 / 17$ in fetuses with SUA (28). In some studies, the dominance of the number of female babies was reported, but different from this rate, it may be 
more or less (29-31). There was no significant difference between the gender ratio in the results of our study. Additional studies are needed to elucidate the role of the sex on chromosomal abnormalities, congenital anomalies, and adverse perinatal outcomes.

The role of environmental or genetic factors in the etiology of SUA remains unknown. The umbilical cord should be assessed by the US in the second trimester of pregnancy, as the vast majority of iSUA patients can be identified with a routine US examination (32). This detection is possible by 1 st trimester (11-14 weeks) and the 2nd-trimester US examination $(33,34)$. In our study group, the diagnosis of SUA was verified by the US at 18-23 weeks. Once SUA is detected, a detailed fetal evaluation should be performed with the US; possible abnormalities should be excluded. Pregnant women should be informed about possible adverse perinatal outcomes after the fetus is diagnosed as iSUA. Close follow up should be performed.

There are some limitations to this study. This study has been designed retrospectively and has the potential to contain limitations of such studies and a relatively low sample size. However, our study would shed light on future studies showing increased perinatal outcomes rates in the iSUA group.

In conclusion, we found a statistically significant low birth weight in the iSUA group. Although oligohydramnios, PPROM, preterm birth, SGA, and NICU admission rates were high, this increase did not reach statistical significance. However, the total adverse perinatal outcome increase was statistically significant. These results indicate that close follow-up is essential to prevent and manage adverse perinatal outcomes in iSUA patients.

Conflicts of interest statement: The authors have no conflicts of interest relevant to this article.

\section{References}

1. Moore KL (1988) The cardiovascular system. In: Moore KL (ed) The Developing Human, 4th edn.Philadelphia, PA: W.B. Saunders Co., pp 286-333.

2. Gornall AS, Kurinczuk JJ, Konje JC. Antenatal detection of a single umbilical artery: does it matter? Prenat Diagn 2003; 23: 117-123.

3. Bombrys AE, Neiger R, Hawkins S, Sonek J, Croom C, McKenna D, et al. Pregnancy outcome in isolate single umbilical artery. Am J Perinatol 2008; 25: 239-242.

4. Wiegand S, McKenna DS, Croom C, Ventolini G, Sonek JD, Neiger R. Serial sonographic growth assessment in pregnancies complicated by an isolated single umbilical artery. Am J Perinatol 2008; 25: 149-152.

5. Battarbee AN, Palatnik A, Ernst LM, Grobman WA. Placental abnormalities associated with isolated single umbilical artery in-small-for-gestational-age births. Placenta 2017; 59: 9-12.

6. Feldstein VA, Harris RD, Machin GA (2008) Ultrasound evaluation of the placenta and umbilical cord. In: Callen PW (ed). Ultrasonography in Obstetrics and Gynecology. 5th edn. Philadelphia, PA: Saunders Elsevier pp 744.

7. Chow JS, Benson CB, Doubilet PM. Frequency and nature of structural anomalies in fetuses with single umbilical arteries. J Ultrasound Med 1998; 17: 765-768.

8. Saller DN, Keene CL, Sun CC, Schwartz S. The association of single umbilical artery with cytogenetically abnormal pregnancies. Am J Obstet Gynecol 1990; 163: 922-925.

9. Sherer DM, Khoury-Collado F, Dalloul M, Osho JA, Lamarque MD, Fomitcheva L, et al. Recurrent antepartum compression of a single artery double nuchal cord necessitating emergency cesarean delivery. Am J Perinatol 2005; 22: 437-440.

10. Kim HJ, Kim J, Chay DB, Park JH, Kim MA. Association of isolated single umbilical artery with perinatal outcomes: Systemic review and meta-analysis. Obstet Gynecol Sci 2017; 60: 266-273.

11. Chetty-John S, Zhang J, Chen Z, Albert P, Sun L, Klebanoff M, et al. Long-term physical and neurologic development in newborn infants with isolated single umbilical artery. Am J Obstet Gynecol 2010; 203: 368e1-7.

12. Horton AL, Barroilhet L, Wolfe HM. Perinatal outcomes in isolated single umbilical artery. Am J Perinatol 2010; 27: 321-324.

13. Hua M, Odibo AO, Macones GA, Roehl KA, Crane JP, Cahill AG. Single umbilical artery and its associated findings. Obstet Gynecol 2010; 115: 930-934.

14. Tulek F, Kahraman A, Taşkın S, Özkavukçu E, Söylemez F. Determination of risk factors and perinatal outcomes of singleton pregnancies complicated by isolated single umbilical artery in Turkish population. J Turk Ger Gynecol Assoc 2015; 16: 21-24.

15. Murphy-Kaulbeck L, Dodds L, Joseph KS, Van den Hof M. Single umbilical artery risk factors and pregnancy outcomes. Obstet Gynecol 2010; 116: 843-850. 
16. Lilja M. Infants with single umbilical artery studied in a national registry. General epidemiological characteristics. Paediatr Perinat Epidemiol 1991; 5: 27-36.

17. Burshtein S, Levy A, Holcberg G, Zlotnik A, Sheiner E. Is single umbilical artery an independent risk factor for perinatal mortality? Arch Gynecol Obstet 2011; 283: 191-194.

18. Dagklis T, Defigueiredo D, Staboulidou I, Casagrandi D, Nicolaides KH. Isolated single umbilical artery and fetal karyotype. Ultrasound Obstet Gynecol 2010; 36: 291-295.

19. Xu Y, Ren L, Zhai S, Luo X, Hong T, Liu R, et al. Association Between Isolated Single Umbilical Artery and Perinatal Outcomes: A Meta-Analysis. Med Sci Monit 2016; 22: 1451 1459.

20. Battarbee AN, Palatnik A, Ernst LM, Grobman WA. Association of Isolated Single Umbilical Artery With Small for Gestational Age and Preterm Birth. Obstet Gynecol 2015; 126: 760-764.

21. Mu S, Lin C, Chen Y, Sung T, Bai C, Jow G. The Perinatal Outcomes of Asymptomatic Isolated Single Umbilical Artery in Full-term Neonates. Pediatr Neonatol 2008; 49: 230-233.

22. Caldas LM, Liao A, Carvalho MH, Francisco RP, Zugaib M. Should fetal growth be a matter of concern in isolated single umbilical artery? Rev Assoc Med Bras (1992) 2014; 60: 125-130.

23. Blum M, Weintraub AY, Baumfeld Y, Rotem R, Pariente G. Perinatal Outcomes of Small for Gestational Age Neonates Born With an Isolated Single Umbilical Artery. Front Pediatr 2019; 7: 79 .

24. Naveiro-Fuentes M, Carrillo-Badillo MP, Malde-Conde J, Gallo-Vallejo JL, PuertasPrieto A. Perinatal outcomes in singleton pregnancies with a single umbilical artery. J Matern Fetal Neonatal Med 2016; 29: 15621565.

25. Ashwal E, Melamed N, Hiersch L, Edel S, Bardin R, Wiznitzer A, et al. The impact of isolated single umbilical artery on labor and delivery outcome. Prenat Diagn 2014; 34: 581 585.

26. Ebbing C, Kessler J, Moster D, Rasmussen S. Isolated single umbilical artery and the risk of adverse perinatal outcome and third stage of labor complications: A population-based study. Acta Obstet Gynecol Scand 2020; 99: 374-380.

27. Thummala MR, Raju TNK, Langenberg P. Isolated Single Umbilical Artery Anomaly and the Risk for Congenital Malformations: A Meta-Analysis. J Pediatr Surg 1998; 33: 580585.

28. Joó JG, Beke A, Papp Z, Rigó J, Papp C. Single umbilical artery in fetopathological investigations. Pathol ResPract 2008; 204: 831-836.

29. Blazer S, Sujov P, Escholi Z, Itai BH, Bronshtein M. Single umbilical artery; right or left? Does it matter? Prenatal Diagn 1997; 17: 5-8.

30. Doornebal N, de Vries TW, Bos AF, De Vries NK. Screening infants with an isolated single umbilical artery for renal anomalies: nonsense? Early Hum Dev 2007; 83: 567-570.

31. Wu MH, Chang FM, Shen MR, Yao BL, Chang $\mathrm{CH}$, Yu CH, et al. Prenatal sonographic diagnosis of single umbilical artery. J Clin Ultrasound 1997; 25: 425-430.

32. Hill LM, Wibner D, Gonzales P, Chenevey P. Validity of transabdominal sonography in the detection of a two-vessel umbilical cord. Obstet Gynecol 2001; 98: 837-842.

33. Rembouskos G, Cicero S, Longo D, Sacchini C, Nicolaides KH. Single umbilical artery at 11-14 weeks' gestation: relation to chromosomal defects. Ultrasound Obstet Gynecol 2003; 22: 567-570.

34. Stout MJ, Odibo AO, Longman R, Shanks AL, Cahill AG. The incidence of isolated single umbilical artery in twins and adverse pregnancy outcomes. Prenat Diagn 2013; 33: 269-272. 